\title{
Two new species of the millipede genus Hypocambala Silvestri, 1895 from China and Vietnam (Diplopoda: Spirostreptida: Cambalopsidae)
}

\author{
Ава новых вида диплопод рода Hypocambala Silvestri, 1895 из \\ Китая и Вьетнама (Diplopoda: Spirostreptida: Cambalopsidae)
}

\author{
S.I. Golovatch ${ }^{1}$, J.-J. Geoffroy ${ }^{2}$, J.-P. Mauriès ${ }^{3} \&$ D. VandenSpiegel ${ }^{4}$ \\ С.И. Головач ${ }^{1}$, Ж.-ЖК. ЖКоффруа ${ }^{2}$ ЖК.-П. Морьес ${ }^{3}$, \\ А. ВанденШпигель ${ }^{4}$
}

\author{
${ }^{1}$ Institute for Problems of Ecology and Evolution, Russian Academy of Sciences, Leninsky pr. 33, Moscow 119071, Russia. \\ ${ }^{1}$ Институт проблем экологии и эволюции РАН, Ленинский пр. 33, Москва 119071 Россия. \\ ${ }^{2}$ Muséum national d'Histoire naturelle, Département Ecologie \& Gestion de la Biodiversité, UMR 7204 CERSP du CNRS, Equipe \\ EVOLTRAIT, 4, avenue du Petit Château, F-91800 Brunoy, France. \\ ${ }^{3}$ Muséum national d'Histoire naturelle, Département Systématique et Evolution, \\ Section Arthropodes, Case Postale n53, 61 rue Buffon, F-75231 Paris Cedex 05, France. \\ ${ }^{4}$ Musée Royal de l'Afrique centrale, B-3080 Tervuren, Belgium.
}

KEY WORDS: Diplopoda, Hypocambala, taxonomy, new species, key, cave, China, Vietnam.

КЛЮЧЕВЫЕ СЛОВА: Diplopoda, Glyphiulus, таксономия, новый вид, ключ, пещера, Китай, Вьетнам.

ABSTRACT. Two new species of the genus $H y$ pocambala are described: H. polytricha sp.n., from a cave in Guangxi Province, China, and H. oligotricha sp.n., from a forest in north-central Vietnam. Both of the new species are especially similar to $H$. vietnamica (Mauriès, 1977), from a cave in Ke Bang karst, northcentral Vietnam, due to the presence of crests/carinae on the collum and following metazona. A key is proposed to separate these three species.

РЕЗЮМЕ. Описаны два новых вида рода Нуроcambala: H. polytricha sp.n. из пещеры в провинции Гуанси (Китай) и H. oligotricha sp.n. из лесной подстилки на северо-востоке Вьетнама. Благодаря наличию ребер на коллуме и следующих метазонитах, оба эти вида особенно похожи на $H$. vietnamica (Maurius, 1977) из пещеры на в карстовом массиве Ке-Банг на северо-востоке Вьетнама. Дан ключ для разделения этих трех видов.

\section{Introduction}

The millipede genus Hypocambala Silvestri, 1895 has relatively recently been reviewed and shown to comprise ten species [Jeekel, 2004]. Only one change has since been made, i.e. the incorporation of $H$. vietnamica (Mauriès, 1977), from a cave in Ke Bang karst, north-central Vietnam [Golovatch et al., 2011].

The genus is mainly characterized by its species showing $O^{7}$ legs 1 more or less walking-leg-like, with the coxae being contiguous and each supplied with an evident distomedian process whose tip in curved forward [Jeekel, 1963; Golovatch et al., 2007, 2011]. Most of the Hypocambala species, which range from the Seychelles, Comoro Islands and Mauritius in the Indian Ocean, through Indochina and the Indo-Australian Archipelago, to New Caledonia, Loyalty Islands, Tonga and Samoa in the Pacific [Jeekel 1963, 2004], lack tergal crests, tergal pilosity being present instead. The single crested congener hitherto known has been $H$. vietnamica. When describing it, Mauriès [1977] chose to place it into Glyphiulus Gervais, 1847, rather than Hypocambala (which is also represented in Vietnam by $H$. gracilis (Attems, 1938)), only because the terga in G. vietnamicus were carinate. Since the carinotaxy patterns in Cambalopsidae tend to be characteristic of species, whereas the structure of $\sigma^{7}$ legs 1 is rather diagnostic for species-groups or genera, we have recently ejected $G$. vietnamicus from the javanicusgroup of Glyphiulus and transferred it to Hypocambala [Golovatch et al., 2011].

Nine of the ten nominate species of Hypocambala, which are strictly juliform and show crests neither on the collum nor on the following metazona, have long been keyed [Jeekel, 1963]. Only one congener, H. monilus (Chamberlin, 1945), from Java, Indonesia, was described too poorly to be incorporated in a key [see Chamberlin, 1945]. More information can be obtained from Jeekel's [2004] catalogue which is still nearly fully relevant concerning Hypocambala.

The present paper puts on record two new species of Hypocambala, both markedly sharing with $H$. vietnamica the presence of carinae on the collum and 

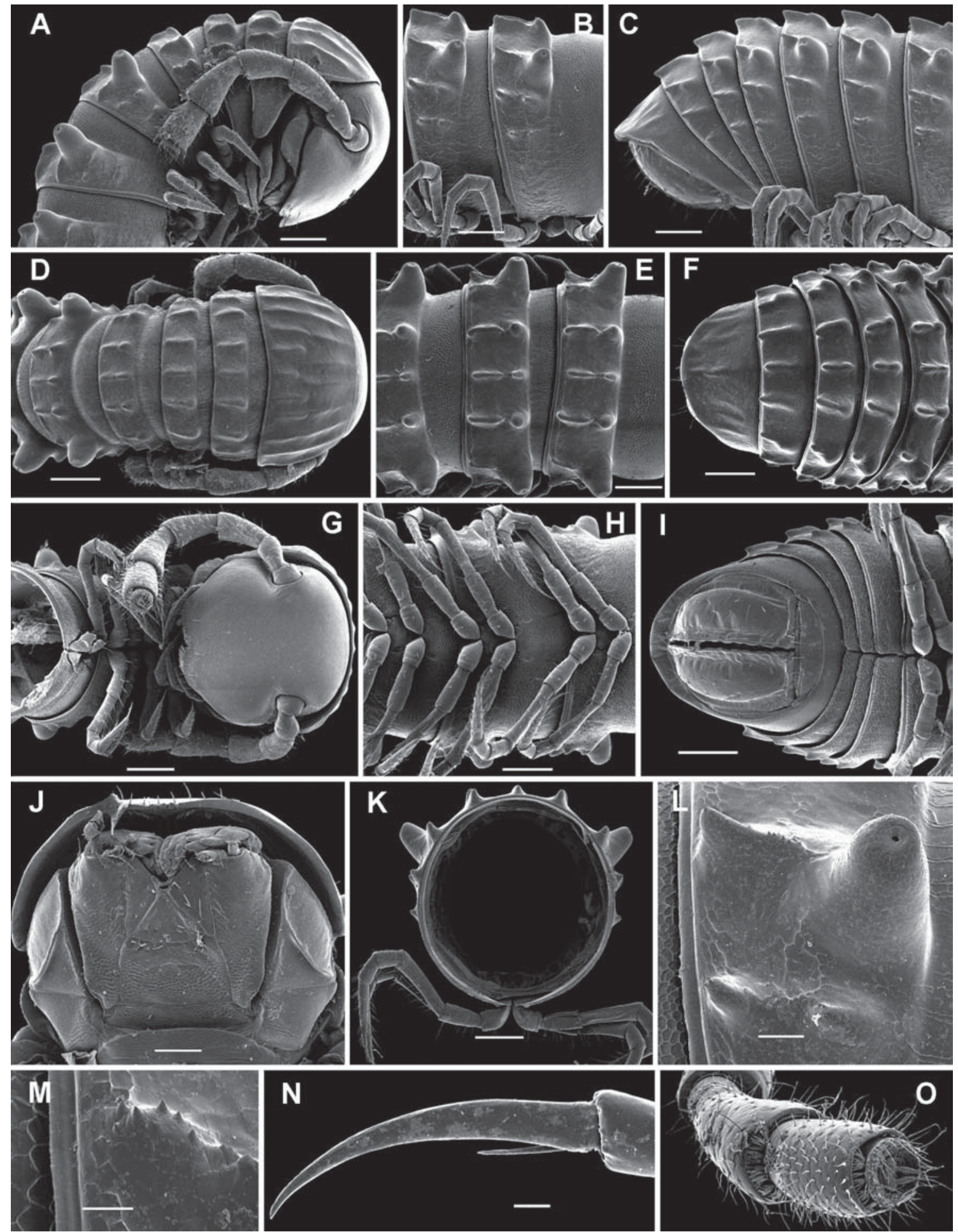

Fig. 1. Hypocambala polytricha sp.n.,, paratype: A, D \& G — anterior part of body, lateral, dorsal and ventral views, respectively; B, E \& H - midbody segments, lateral, dorsal and ventral views, respectively; C, F \& I - posterior part of body, lateral, dorsal and ventral views, respectively; J - gnathochilarium, ventral view; K - cross-section of a midbody segment, caudal view; L - enlarged ozopore region, lateral view; $\mathrm{M}$ - enlarged crest in front of ozoporiferous cone, lateral view; $\mathrm{N}$ - enlarged claw, lateral view; $\mathrm{O}$ - distal part of antenna, dorsocaudal view. Scale bars: A-I, K-0.2 mm; J-0.1 mm; L, O $-0.05 \mathrm{~mm} ; \mathrm{M}-0.02 \mathrm{~mm} ; \mathrm{N}-0.01 \mathrm{~mm}$.

Рис. 1. Hypocambala polytricha sp.n., паратип +: A, D, G - передняя часть тела, соответственно сбоку, сверху и снизу; В, Е, H - среднетуловищные сегменты, соответственно сбоку, сверху и снизу; C, F, I - задняя часть тела, соответственно сбоку, сверху и снизу; J — гнатохилярий, снизу; K — поперечный срез через среднетуловищный сегмент, сзади; L — увеличенный район озопоры, сбоку; М - увеличенное ребро впереди несущего озопору конуса, сбоку; $\mathrm{N}$ - увеличенный коготок, сбоку; О дистальная часть антенны, сверху и сзади. Масштаб: A-I, $\mathrm{K}-0,2$ мм; J - 0,1 мм; L, O - 0,05 мм; M - 0,02 мм; N-0,01 мм. 


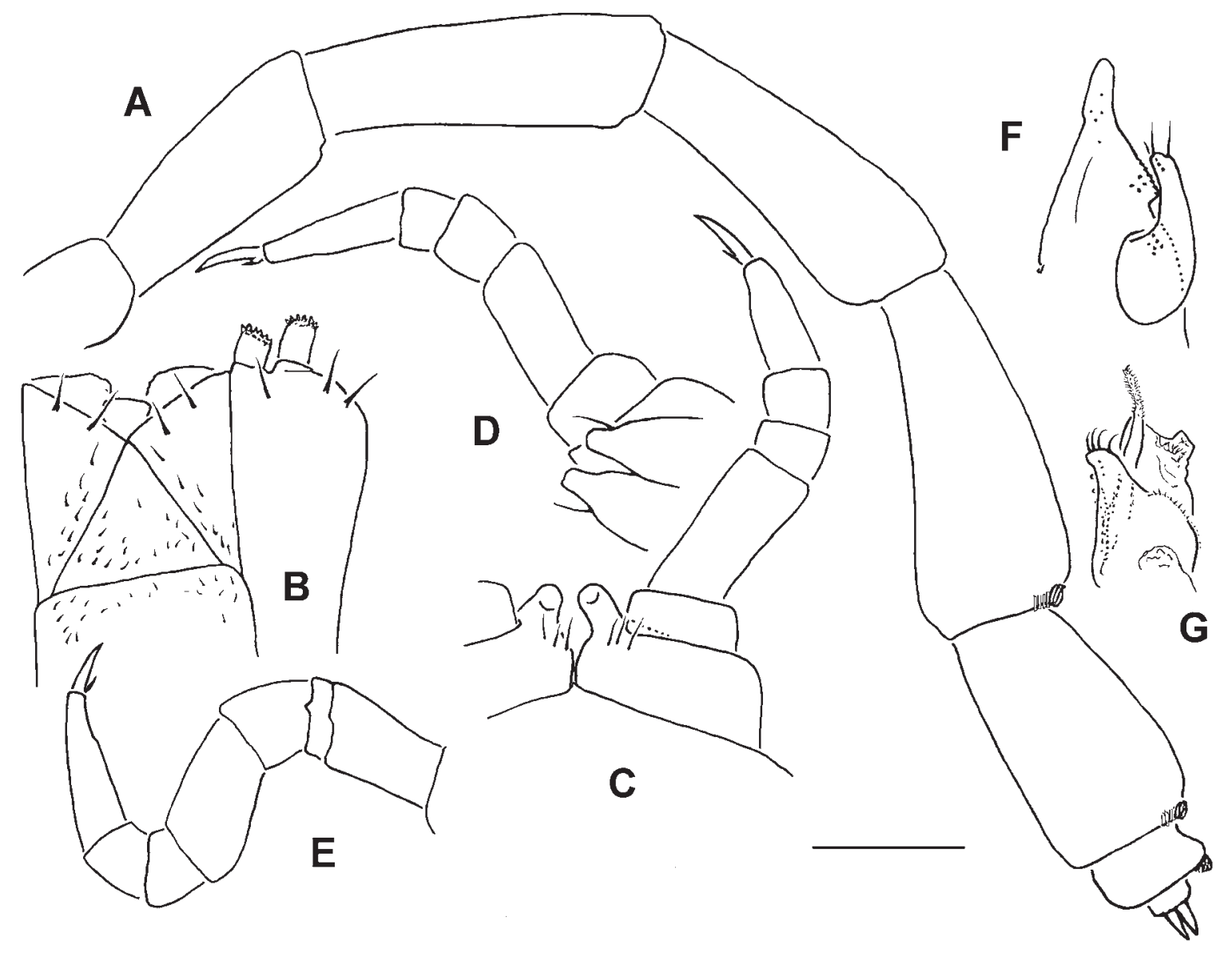

Fig. 2. Hypocambala polytricha sp.n., holotype: A - antenna, lateral view; B - gnathochilarium, ventral view; C - legs 1 , front view; D - leg 2, caudal view; E - leg 3, caudal view; F — left anterior gonopod, caudal view; G - left posterior gonopod, caudal view. Scale bar: $0.2 \mathrm{~mm}$.

Рис. 2. Hypocambala polytricha sp.n., голотип: А — антенна, сбоку; В — гнатохилярий, снизу; С — ноги 1, сзади; D — нога 2, сзади; Е — нога 3, сзади; F — левый передний гонопод, вид сзади; G — левый задний гонопод, вид сзади. Масштаб: 1,0 мм.

following metazona. A key is also proposed to separate these three species.

Abbreviations used:

IZAS - Institute of Zoology, Academia Sinica, Beijing, China;

MNHN - Muséum national d'Histoire naturelle, Paris, France;

SCAU — South China Agricultural University, Guangzhou, China;

SEM - Scanning electron microscopy.

\section{Material and methods}

The material serving as the basis for the present contribution derives from the material taken in China and Vietnam by Anne Bedos and Louis Deharveng (MNHN). This material has been shared between the collections of MNHN, IZAS and SCAU, as indicated hereafter.
SEM micrographs were taken using a JEOL JSM6480LV scanning electron microscope. After examination, SEM material was removed from stubs and returned to alcohol, all such samples being kept at MNHN.

\section{Taxonomic part \\ Hypocambala polytricha sp.n. Figs $1 \& 2$.}

MATERIAL. Holotype $\mathrm{O}^{7}$ (IZAS), China, Guangxi Prov., Longzhou County, Nonggang, Cave Bi Ji Dong (Ca Wai), $22.67402^{\circ} \mathrm{N}$, $106.76977^{\circ} \mathrm{E}$, hand collection, 08.03.2005, leg. L. Deharveng \& A. Bedos (CHIgx05-072). Paratypes: 1 \% juv. (SCAU), 1 \% (SEM), same locality, together with holotype.

NAME. To emphasize the highly polytrichous gnathochilarium.

DIAGNOSIS. Differs from crested congeners in the polytrichous gnathochilarium, coupled with the ab- 

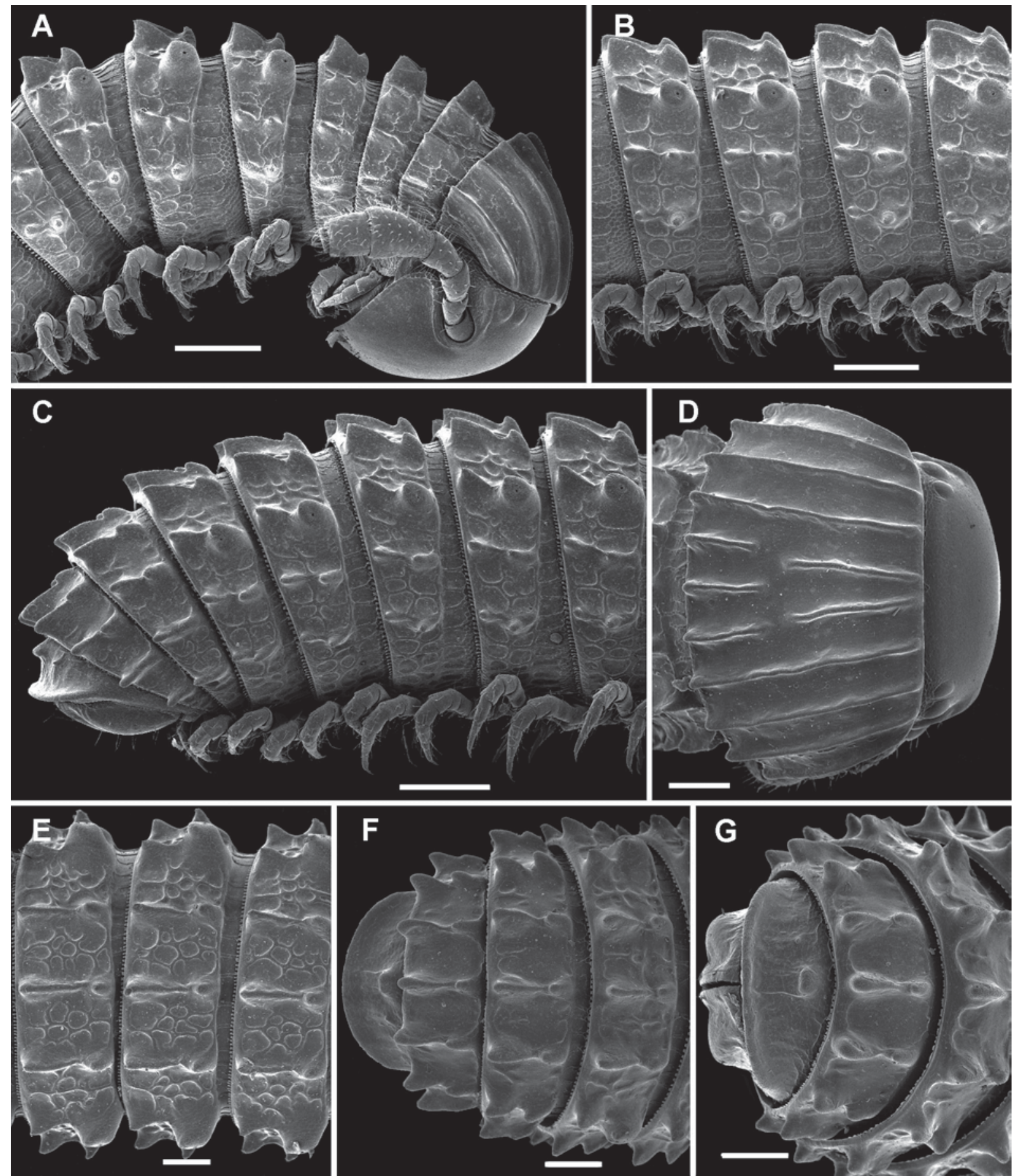

Fig. 3. Hypocambala oligotricha sp.n., juvenile $\mathrm{O}^{T}$ paratype: A \& D — anterior part of body, lateral and dorsal views, respectively; B \& E - midbody segments, lateral and dorsal views, respectively; C, F \& G - caudal part of body, lateral, dorsal and caudal views, respectively. Scale bars: A-C $-0.2 \mathrm{~mm} ; \mathrm{D}-\mathrm{G}-0.1 \mathrm{~mm}$.

Рис. 3. Hypocambala polytricha sp.n., ювенильный паратип О : A, D - передняя часть тела, соответственно сбоку и сверху; В, Е - среднетуловищные сегменты, соответственно сбоку и сверху; C, F, G - задняя часть тела, соответственно сбоку, сверху и сзади. Масштаб: $\mathrm{A}-\mathrm{C}-0,2 \mathrm{мm} ; \mathrm{D}-\mathrm{G}-0,1$ мм.

sence of ocelli, the peculiar shape of the epiproct etc. (see also Key below).

DESCRIPTION. Length of holotype ca $32 \mathrm{~mm}$, width $1.7 \mathrm{~mm}$, with $52 \mathrm{p}+1 \mathrm{a}+\mathrm{T}$. Coloration uniformly pallid to light yellow-brown; crests on collum and segments 2-4 slightly infuscate, light brown, while ozoporiferous cones sometimes brown.

Ocelli completely reduced. Antennae long and slender (Figs 1A, G, O, 2A), extending beyond segment 4 laterally, antennomeres 5-7 each with a rather large 

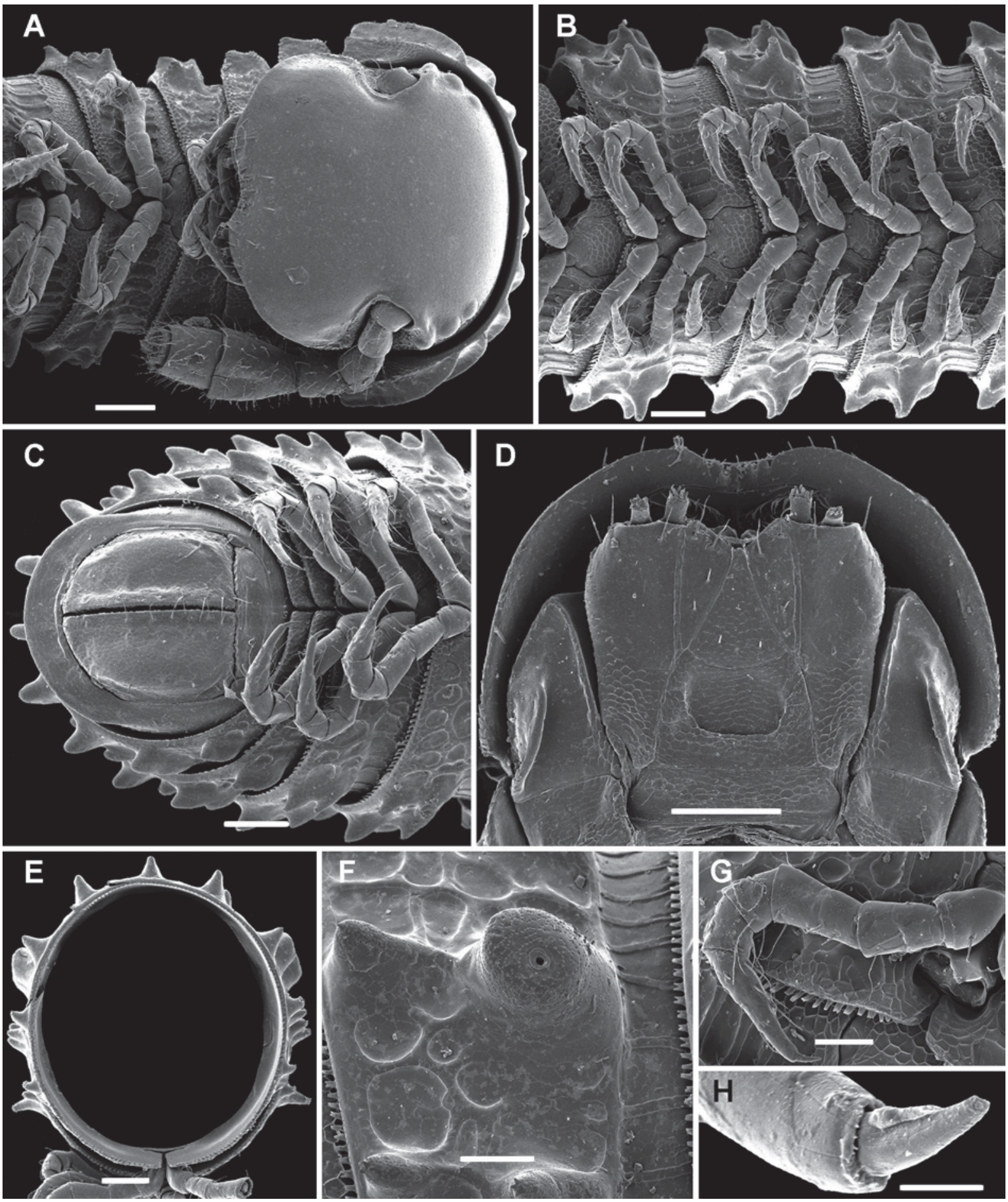

Fig. 4. Hypocambala oligotricha sp.n., juvenile $0^{7}$ paratype: A — anterior part of body, ventral view; B - midbody segments, ventral view; C — caudal part of body, ventral view; D — gnathochilarium, ventral view; E — cross-section of a midbody segment, caudal view; $\mathrm{F}$ - enlarged ozopore region and limbus, lateral view; $\mathrm{G}$ - midbody leg, front view; $\mathrm{H}$ - enlarged claw, ventrolateral view. Scale bars: A-E $-0.1 \mathrm{~mm} ; \mathrm{F}, \mathrm{G}-0.05 \mathrm{~mm} ; \mathrm{H}-0.01 \mathrm{~mm}$.

Рис. 4. Hypocambala oligotricha sp.n., ювенильный паратип О7: А - передняя часть тела, снизу; В - среднетуловищные сегменты, снизу; С — задняя часть тела, снизу; D — гнатохилярий, снизу; Е - поперечный разрез через среднетуловищный сегмент, сзади; F - увеличенный район озопоры и лимбус, сбоку; $\mathrm{G}$ - среднетуловищная нога, спереди; Н - увеличенный коготок, снизу и сбоку. Масштаб: А-E - 0,1 мм; F, G-0,05 мм; H - 0,01 мм. 
distodorsal group of bacilliform sensilla. Gnathochilarium with a separate promentum, polytrichous (Figs 1J, 2B) $(\mathrm{n}=2)$.

Postcollum constriction moderate. Crests on collum mostly evident, only mid-dorsally slightly flattened/obliterate (Figs 1A, D). Carinotaxy formula of collum, I-IV $+5 \mathrm{a}+\mathrm{pc}+\mathrm{ma}$.

Subsequent metaterga strongly crested (Figs $1 \mathrm{~A}-\mathrm{F}$, $\mathrm{K}$ ); carinotaxy formula, $2 / 2+\mathrm{I} / \mathrm{i}+3 / 3+\mathrm{I} / \mathrm{i}+2 / 2$. Ozoporiferous tubercles especially large, conical, directed anterolaterad (like all others), elongated on top, higher than wide; midbody metatergal crests shorter, divided into two at about $1 / 3^{\text {rd }}$ extent, front part especially high and sharp (Figs 1A-F), crest in front of ozoporiferous cone microdentate (Figs 1L, M). Midbody segments slightly compressed in cross-section (Fig. 1K).

Tegument finely alveolate-areolate, dull throughout. Fine longitudinal striations in front of stricture between pro- and metazona, remaining surface of prozona very delicately shagreened. Segments 2 and 3 with long pleural flaps (Fig. 1A). Limbus extremely finely and regularly microdenticulate, nearly entire. Epiproct (Figs 1C, F) simple, regularly rounded caudally, only slightly rugulose dorsally; caudal margin straight, not upturned. Paraprocts rather regularly convex (Fig. 1I), median margins only slightly elevated due to premarginal sulci. Hypoproct transversely lanceolate (Fig. 1I).

Ventral flaps behind gonopod opening on segment 7 barely distinguishable as low swellings, not forming a marked transverse ridge.

Legs long and slender (Figs 1H, K), on midbody segments 1.2-1.3 length of segment height. Claw at base with an evident accessory spine less than half as long as claw itself (Fig. 1N).

$\sigma^{7}$ legs 1 (Fig. 2C) complete; coxae contiguous, each with a distomedian process curved anteriorly on top. $O^{7}$ legs 2 (Fig. 2D) not hypertrophied; penes bottle-shaped, each with a strong seta distolaterally. $\sigma^{7}$ legs 3 (Fig. 2E) slightly modified in having coxa faintly elongate.

Anterior gonopods (Fig. 2F) with a typical shieldlike coxosternum, this being high, rather abundantly microsetose on caudal face, provided with a conspicuous, high, terminally axe-shaped, mesal process. Telopodite small but movable, 1-segmented, lateral in position, with three strong apical setae and a field of microscopic setae/cones at base, considerably longer than adjacent, finely serrate, lateral corner of coxosternum. Posterior gonopods (Fig. 2G) rather compact, coxite with a long, medio-apical, pilose flagellum and a hyaline, membranous, apicolateral lobule.

REMARKS. The absence of ocelli and coloration, as well as the long appendages might be evidence of $H$. polytricha sp.n. being a troglobiont.

\section{Hypocambala oligotricha sp.n.}

Figs 3-5.

MATERIAL. Holotype $\sigma^{7}$ (MNHN GA 092), Vietnam, Quang Binh Prov., Cha Noi Distr., Cha Noi, $17^{\circ} 38^{\prime} 0^{\prime \prime} \mathrm{N}, 106^{\circ} 7^{\prime} 0^{\prime \prime} \mathrm{E}$, forest litter, Berlese extraction, 08.01.1995, leg. L. Deharveng \& A. Bedos (VIET-078). Paratypes: $1 \sigma^{7}$ (MHNH GA 092), $1 \sigma^{7}$ juv. (SEM), same locality, together with holotype.

NAME. To emphasize the oligotrichous gnathochilarium.

DIAGNOSIS. Differs from crested congeners in the oligotrichous gnathochilarium, coupled with a vivid colour pattern, the peculiar shape of the epiproct etc. (see also Key below).

DESCRIPTION. Length of holotype ca $10 \mathrm{~mm}$, width $0.8 \mathrm{~mm}$, with $32 \mathrm{p}+3 \mathrm{a}+\mathrm{T}$. Paratype $\mathrm{O}^{7}$ ca $14 \mathrm{~mm}$ long and $0.9 \mathrm{~mm}$ wide, with $40 \mathrm{p}+1 \mathrm{a}+\mathrm{T}$. Coloration vividly variegated, due to pallid to light yellow head, segments 2 and 3, and venter, a basically light yellow(-brownish) dorsum, brown legs, dark brown antennae, ocellaria, anterior halves of crests on collum and both parts of crests on metaterga, and, above all, distinct, rather broad, continuous, lateral, marbled brown stripes level to ozoporiferous cones starting from segment 4.

A few (3) dark brown ocelli in a single vertical row on each side of head (Figs 3A, D, 4A). Antennae short and stout (Figs 3A, 4A, 5A), extending slightly beyond segment 3 laterally, antennomeres 5 and 6 each with a rather small distodorsal group of bacilliform sensilla. Gnathochilarium with a separate promentum, oligotrichous (Figs 4D, 5B) $(\mathrm{n}=2)$.

All crests on collum evident (Figs 3A, D). Carinotaxy formula of collum, I-IV $+5 \mathrm{a}+\mathrm{pc}+\mathrm{ma}$.

Subsequent metaterga strongly crested (Figs 3, 4E); carinotaxy formula, $2 / 2+\mathrm{I} / \mathrm{i}+3 / 3+\mathrm{I} / \mathrm{i}+2 / 2$. Ozoporiferous tubercles especially large, conical, directed caudolaterad (like all others), stout, wider than high; midbody metatergal crests barely shorter, divided into two at about $1 / 3^{\text {rd }}$ extent, both parts being high and sharp; no microdenticles on crest in front of ozoporiferous cone (Figs 4E, F). Midbody segments slightly compressed in cross-section (Fig. 4E).

Tegument finely alveolate-areolate, dull throughout (Fig. 4F). Fine longitudinal striations in front of stricture between pro- and metazona, remaining surface of prozona very delicately shagreened. Segments 2 and 3 with long pleural flaps (Fig. 3A). Limbus rather evidently and regularly microdenticulate (Fig. 4F). Epiproct (Fig. 3C, F, G) simple, regularly rounded caudally with an evident central tooth at base dorsally; caudal margin slightly upturned. Paraprocts rather regularly convex (Figs 3C, G), median margins only slightly elevated due to premarginal sulci (Fig. 4C). Hypoproct transversely lanceolate, caudal margin very finely microdenticulate (Fig. 4C).

Ventral flaps behind gonopod opening on segment 7 barely distinguishable as low swellings, not forming a marked transverse ridge.

Legs rather short and stout (Figs 4B, E, G, 5F), on midbody segments $2 / 3$ rds length of segment height. Claw at base with an evident accessory spine only a little less than half as long as claw itself (Fig. 4H).

$\sigma^{7}$ legs 1 (Fig. 5C) complete; coxae contiguous, each with a distomedian process curved anteriorly on 


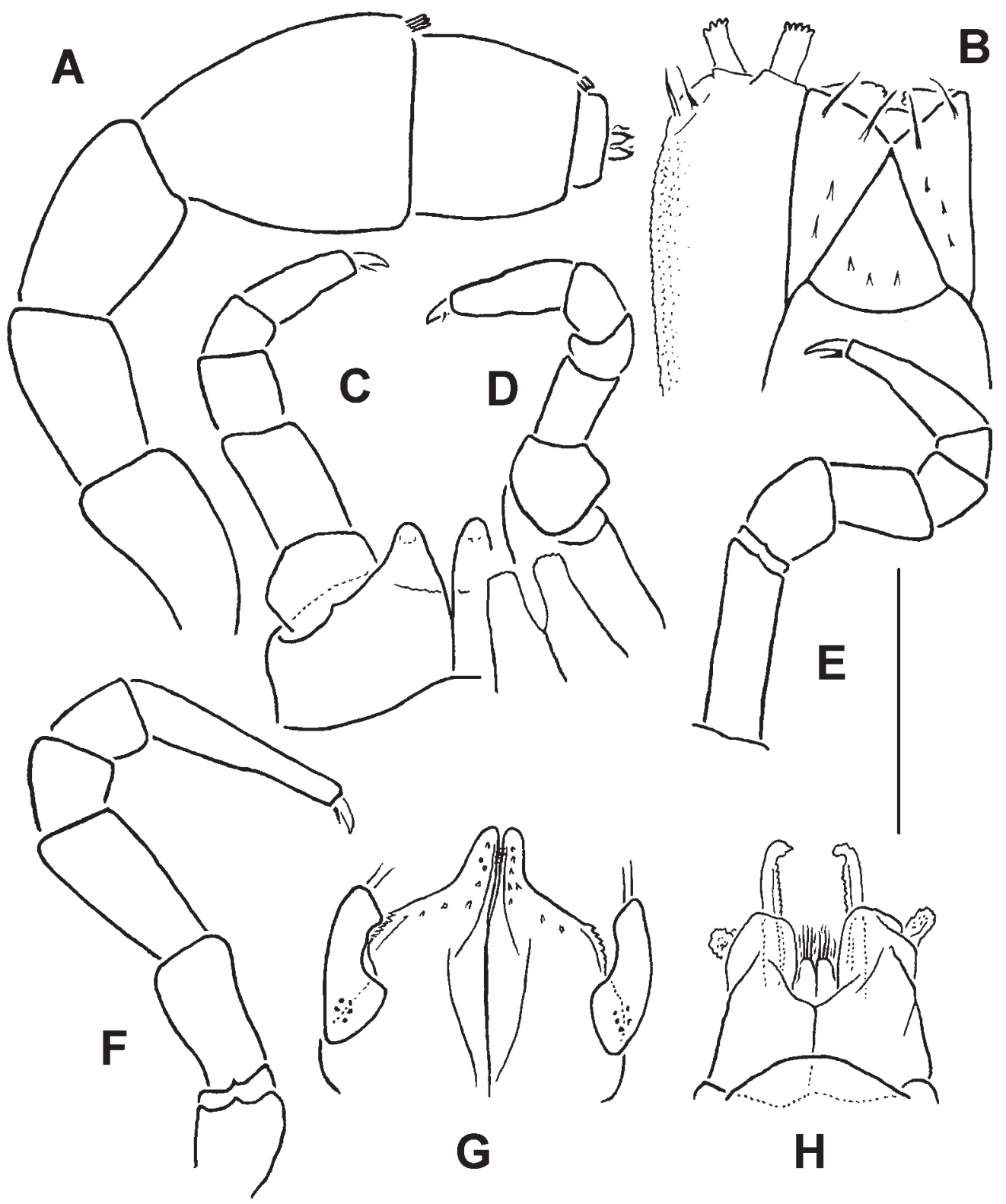

Fig. 5. Hypocambala oligotricha sp.n., $\mathrm{O}^{7}$ paratype: A — antenna, lateral view. B — gnathochilarium, ventral view; C - legs 1, front view; D - leg 2, caudal view. E — leg 3, caudal view; F — midbody leg, lateral view; G - anterior gonopods, caudal view; H - posterior gonopods, caudal view. Scale bar: A-F $-0.4 \mathrm{~mm}$;, $\mathrm{H}-0.2 \mathrm{~mm}$.

Рис. 5. Hypocambala oligotricha sp.n., паратип О?: А - антенна, вид сбоку; В — гнатохилярий, вид снизу; С — ноги 1, спереди; D - нога 2, сзади; Е - нога 2, сзади; F - среднетуловищная нога, сбоку; G - передние гоноподы, сзади; Н - задние гоноподы, сзади. Масштаб: А-F - 0,4 мм; G, H - 0,2 мм.

top. $\bigcirc^{7}$ legs 2 (Fig. 5D) not hypertrophied; penes linguiform, bare. $\sigma^{7}$ legs 3 (Fig. 5E) modified in having coxa considerably elongate.

Anterior gonopods (Fig. 5G) with a typical shieldlike coxosternum, this being high, rather abundantly microsetose on caudal face, provided with a conspicuous, high, terminally finger-shaped, mesal process. Telopodite small but movable, 1-segmented, lateral in position, with two strong apical setae and a field of microscopic setae/cones at base, considerably longer than adjacent, finely serrate, lateral corner of coxosternum. Posterior gonopods (Fig. 5H) rather compact, coxite with a long, medio-apical, only slightly rugulose/serrate flagellum and a hyaline, membranous, apicolateral lobule.

\section{Key to CRESTED HyPOCAMBALA SPECIES}

1. Carinotaxy formula of collum: $\mathrm{I}-\mathrm{V}+\mathrm{ma}$. Epiproct with $1+1$ small knobs dorsally H. vietnamica 
- Carinotaxy formula of collum: I-IV $+5 a+p c+m a$ (Figs 1A, D, 3A, D). Dorsal surface of epiproct either rugulose or with a central knob at base

2. Colour pattern absent, coloration uniformly pallid to yellow. Antennae long and slender, extending behind segment 4 in lateral view (Fig. 1A). Ocelli completely reduced (Figs 1A, G). Gnathochilarium polytrichous (Figs 1J, 2B). Crests on collum somewhat flattended/obliterate mid-dorsally (Figs 1A, D). Dorsal surface of epiproct rugulose (Fig. 1F). A cave in Guangxi Prov., China ..... H. polytricha sp.n.

- Colour pattern distinct, especially so due to brown ocellaria, antennae, legs, tergal crests and two broad lateral stripes level to ozoporiferous cones. Antennae short and clavate, extending behind segment 3 in lateral view (Figs 3A, 5A). Ocelli (3) arranged in a transverse row (Figs 3A, D, 4A). Gnathochilarium oligotrichous (Figs 4D, 5B). All crests on collum fully developed (Figs 3A, D). Epiproct with a central knob dorsally at base (Figs 3C, F, G). Forest litter in northeastern Vietnam

$$
\text { H. oligotricha sp.n. }
$$

ACKNOWLEDGEMENTS. This work only became possible through the support offered to the first author by the Muséum national d'Histoire naturelle, Paris. Both Louis Deharveng and Anne Bedos (both MNHN) are deeply thanked for the precious material they provided for study.

\section{References}

Chamberlin R.V. 1945. On some diplopods from the Indo-Australian Archipelago // American Museum Novitates. No.1282. P.1-43.

Golovatch S.I., Geoffroy J.-J., Mauriès J.-P., VandenSpiegel D. 2007. Review of the millipede genus Glyphiulus Gervais, 1847 , with descriptions of new species from Southeast Asia (Diplopoda, Spirostreptida, Cambalopsidae). Part 2: the javanicus-group // Zoosystema. Vol.20. No.4. P.417-456.

Golovatch S.I., Geoffroy J.-J., Mauriès J.-P., VandenSpiegel D. 2011. New species of the millipede genus Glyphiulus Gervais, 1847 from the javanicus-group (Diplopoda: Spirostreptida: Cambalopsidae) // Arthropoda Selecta. Vol.20. No.3. P.149165.

Jeekel C.A.W. 1963. Diplopoda of Guiana (1-5) // Studies on the Fauna of Suriname and other Guyanas. Vol.4. No.11. P.1-157.

Jeekel C.A.W. 2004. A bibliographic catalogue of the "Cambaloidea" (Diplopoda, Spirostreptida) // Myriapod Memoranda. Vol.7. P.43-109.

Mauriès J.-P. 1977. Le genre Glyphiulus Gervais, 1847, et sa place dans la classification des Cambalides, à propos de la description d'une nouvelle espèce du Viêt-Nam (Diplopoda, Iulida, Cambalidea) // Bulletin du Muséum national d'Histoire naturelle, 3e sér. No.431 (Zool. 301). P.243-250.

Responsible editor K.G. Mikhailov 